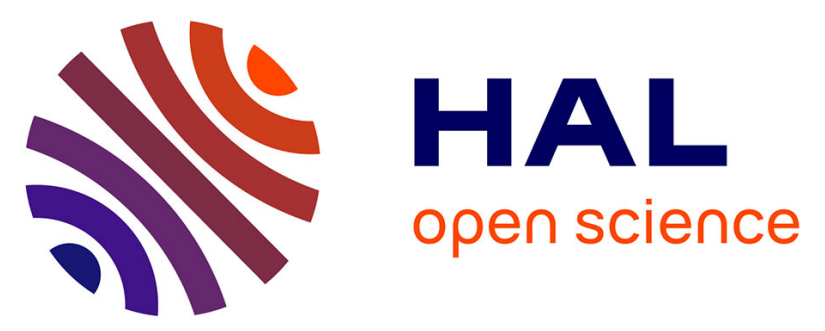

\title{
Conductance of disordered semiconducting nanowires and carbon nanotubes: a chain of quantum dots
} \author{
J.-F. Dayen, T.L. Wade, G. Rizza, D.S. Golubev, C.-S. Cojocaru, D. Pribat,
} X. Jehl, M. Sanquer, J.-E. Wegrowe

\section{- To cite this version:}

J.-F. Dayen, T.L. Wade, G. Rizza, D.S. Golubev, C.-S. Cojocaru, et al.. Conductance of disordered semiconducting nanowires and carbon nanotubes: a chain of quantum dots. European Physical Journal: Applied Physics, 2009, 48 (1), pp.1-14. 10.1051/epjap/2009132 . hal-00505543

\section{HAL Id: hal-00505543 \\ https://hal.science/hal-00505543}

Submitted on 24 Jul 2010

HAL is a multi-disciplinary open access archive for the deposit and dissemination of scientific research documents, whether they are published or not. The documents may come from teaching and research institutions in France or abroad, or from public or private research centers.
L'archive ouverte pluridisciplinaire HAL, est destinée au dépôt et à la diffusion de documents scientifiques de niveau recherche, publiés ou non, émanant des établissements d'enseignement et de recherche français ou étrangers, des laboratoires publics ou privés. 


\title{
Conductance of Disordered Semiconducting Nanowires and Carbon Nanotubes: a Chain of Quantum Dots.
}

\author{
J.-F. Dayen, T. L. Wade, G. Rizza and J.-E. Wegrowe* \\ Laboratoire des Solides Irradiés, Ecole Polytechnique, 91128 Palaiseau Cedex, France. \\ C. Cojocaru, D. Pribat \\ Laboratoire de Physique des interfaces et couches minces, \\ Ecole Polytechnique, 91128 Palaiseau, France. \\ X. Jehl, M. Sanquer \\ CEA-Grenoble, DRFMC/SPSMS/LaTEQS, \\ 17 rue des Martyrs, F-38054 Grenoble cedex 09, France. \\ D. S. Golubev \\ Forschungszentrum Karlsruhe, Institut fur Nanotechnologie, 76021 Karlsruhe, Germany. \\ (Dated: May 7, 2009)
}




\begin{abstract}
A comparative study of the law temperature conductivity of an ensemble of multiwall carbon nanotubes and semiconductor nanowires is presented. The quasi one-dimensional samples are made in nanoporous templates by electrodeposition and CVD growth. Three different structures are studied in parallel: multiwall carbon nanotubes, tellurium nanowires, and silicon nanowires. It is shown that the Coulomb blockade regime dominates the electronic transport below $50 \mathrm{~K}$, together with weak and strong localization effects. In the Coulomb blockade regime, a scaling law of the conductance measured as a function of the temperature and the voltage is systematically observed. This allows a single scaling parameter $\alpha$ to be defined. This parameter accounts for the specific realization of the "disorder", and plays the role of a fingerprint for each sample. Correlations between $\alpha$ and the conductance measured as a function of temperature and voltage, as a function of the perpendicular magnetic field, and as a function of the temperature and voltage in the localized regime below $1 \mathrm{~K}$ have been performed. Three universal laws are reported. They relate the coefficient $\alpha(1)$ to the normalized Coulomb blockade conductance $G_{T}(\alpha),(2)$ to the phase coherence length $l_{\phi}(\alpha)$, and (3) to the activation energy $E_{a}(\alpha)$. These observations suggest a description of the wires and tubes in terms of a chain of quantum dots; the wires and tubes break into a series of islands. The quantum dots are defined by conducting islands with a typical length on the order of the phase coherence length separated by poorly conducting regions (low density of carriers or potential barriers due to defects). A corresponding model is developed in order to put the three universal laws in a common frame.
\end{abstract}

PACS numbers: 72.15.R, 72.20.Ee, 73.23.Hk, 73.63.Fg

*Electronic address: jean-eric. wegrowe@polytechnique.fr 


\section{INTRODUCTION}

The study of electronic properties of nanowires or nanotubes is an active field of research and many questions are still open regarding the reproducibility of the transport parameters from one sample to the other and consequently the ability to massively integrate such objects into efficient nanoelectronic devices. Since a couple of vacancies or a single ion are able to trap electrons, a good single electron transistor should be controlled at the scale of any topological defects and any inhomogeneous charge distribution. However, the disorder is often used in order to create, naturally and randomly, a chain of quantum dots [1]. The reason usually invoked $[1,2]$ is that small defects (topological, chemical, etc) induce strong charge inhomogeneities. The consequence is that some small highly conducting regions $(\mathrm{Q}$. dots) are separated by poorly conducting ones (tunnel barriers). Pictures of such chain of quantum dots have been nicely imaged by scanning probe techniques [3-5]. The aim of this paper is to report a set of empirical laws observed about transport properties in such nanostructures, and to interpret them within the model of a chain of quantum dots.

In disordered nanostructures, the role of defects is not easy to measure since the thermodynamic limit is not necessarily reached for a given sample. Important quantitative variations of the transport parameters are usually observed from one sample to the other even if samples are produced within a well-defined fabrication process [3, 6-16]. Since one sample is a specific realization of a statistical distribution of defects (called "disorder"), the whole distribution can be known by measuring a statistical ensemble of samples. In the study reported here, we use a template synthesis technique [17], in order to produce large enough numbers of samples with different materials. Multi-wall carbon nanotubes (CNT), Te nanowires, and Si nanowires are studied in parallel. Some controlled fabrication parameters (sizes, nature of the wire, nature of the contacts) have been modified, and the variations are hence added to the unknown distribution of structural topological and chemical disorder defined over the ensemble of samples. The goal is to access the statistical distribution of the electronic transport properties, in order to extract some general behavior related to disorder.

Fortunately, in the case of carbon nanotubes (see for example [18-24]), some of the main physical properties have been intensively studied in the last decade. In the present study, these properties (see below) are systematically observed, not only for CNTs, but for all samples of the statistical ensemble. Three main effects are observed and described : (1) 
Coulomb blockade effects, (2) weak localization like effects and (3) transition to an activated regime at low temperatures.

(1) Due to the small diameter, the disorder and the low carrier density, the screening of the electronic charges is poor, and Coulomb interaction occurs between carriers. This Coulomb blockade effect is observed and quantified experimentally through the so-called "zero bias anomaly" profile of the conductance measured as a function of the bias voltage for various temperatures (Fig 3). A scaling law is observed (the effect of the bias voltage scales with that of the temperature) that allows a scaling coefficient $\alpha$ to be measured. Each sample is described by a single parameter $\alpha$, which plays the role of a fingerprint.

(2) The weak-localization effect (or an analogous effect) is also observed systematically in carbon nanotubes and nanowires [14, 23, 25-37]. Weak localization (WL) is a wellknown effect [38-40] due to the interference of an electron with itself during a random walk, and is described in terms of perturbative quantum correction to the Drude conductivity. The main parameter that governs the WL effect, the phase coherence length $l_{\phi}$, can be measured by applying a perpendicular magnetic field to the sample. However, the presence of WL (perturbative correction to the conductivity) together with Coulomb blockade (nonperturbative treatment needed) is puzzling, and this question will also be addressed in this paper. The coefficient $l_{\phi}$ is also correlated to $\alpha$ and shows a second universal behaviour (Sec. III).

(3) Finally, the behavior of the conductivity at very low temperatures (typically below $1 \mathrm{~K}$ ) shows an abrupt transition to a strong-localization like effect where the conductivity is driven by an activation process. The activation process allows another well-defined parameter to be measured which is the activation energy $E_{a}$. It is shown that this transition, observed as a function of temperature, is destroyed by applying a critical bias voltage $V_{c}([41])$, and the Coulomb blockade regime is recovered at higher voltage. The parameters $E_{a}$ and $V_{c}$ are also correlated to the coefficients $\alpha$ (Sec. IV).

After presenting the sample fabrication in Sec II, the three kinds of measurements described above are reported and the corresponding empirical laws are discussed. The challenge is to find a common picture able to describe the three types of the observed behaviors. The simplest picture of Coulomb blockade is given for a system composed by a small Coulomb island separated by two capacitive junctions. The conduction electrons have to overcome the Coulomb energy due to the electric charging of the island. Beyond this, however, some more 
general approaches have been developed in order to describe Coulomb blockade through tunnel barriers in disordered environment. Indeed, generalization of the concept has been developed in the context of single electron devices [42], extended junctions [43], disordered systems [44], and multiwall CNTs [45]. In this picture, the active part of the device is a tunnel junction connected to the rest of a circuit. During the tunnelling process, the electron, electrically screened in the first contact, should recover a screening cloud after tunnelling, and this process costs an amount of energy defined by the impedance of the rest of the circuit. In a more realistic picture (according to previous observations [3-5]), due to charge inhomogeneities within the wire or tube, the quasi 1D system is broken into conducting islands, or quantum dots, separated by poorly conducting regions, or potential energy barriers. In this case, WL effect occurs inside the quantum dots, and Coulomb blockade is generated at the interfaces between the islands and averaged over many junctions. The consequences of the chain of quantum dots, in terms of transport properties, are described formally in the last section ( cf. Section.VI) in relation with the observed effects.

\section{SAMPLE FABRICATION}

The samples are obtained by the template synthesis method in which the growth of the wires is performed inside the pores of a membrane. The diameter of the wires is calibrated by the diameter of the pores (typically around $50 \mathrm{~nm}$ ), and the length is fixed by the thickness of the nanoporous membrane (typically adjusted at $1 \mu \mathrm{m}$ ). A first electric contact is obtained on the bottom through the nucleation process in the electrolytic bath, and the second contact is obtained on the top of the membrane with the help of a second patterned metallic layer.

We will discuss three types of samples: Tellurium nanowires(TeNW) grown and contacted by electrodeposition (see Fig. 1), silicon nanowires ( $\mathrm{Si}$ NW) and multiwall carbon nanotubes (MWCNT) grown with CVD on electrodeposited catalysts (see Fig. 2). All samples are obtained with bottom-up growth inside a nanoporous insulating membrane. The MWCNT were obtained by chemical vapour deposition (thermal CVD) of acetylene at $640^{\circ} \mathrm{C}$, after the electrodeposition of $\mathrm{Ni}$ or Co catalyst inside the nanopores of an alumina membrane (a precise description of the process is given in $[17,22])$. The Si NW are also obtained with thermal CVD of Silane $\left(\mathrm{Si} \mathrm{H}_{4}\right)$ in a $5 \%$ hydrogen atmosphere (a description can be found in $[37])$. 
The first set of samples is composed of single contacted tellurium nanowires -TeNWobtained by electrodeposition in nanoporous polycarbonate or alumina membranes of diameter $\mathrm{d}=40 \mathrm{~nm}$ and $\mathrm{d}=150 \mathrm{~nm}$ and a length of $6 \mu \mathrm{m}$. Fig. 1 (b) shows a zoom of the wire after dissolution of the membrane. The wires are polycrystalline (Fig. 1 (b)) with small disorientations between grains (Fig. 1 (c)). No other defects have been observed. With the electrodeposition technique [17], a single nanowire can be contacted in situ with a feedback loop on the intermembrane electric potential, after depositing a thin enough Au layer on the top. If the electrodeposition potential is maintained, dendritic growth are observed on the surface of the membrane (Fig. $1 \mathrm{~d}$ )). This is the typical form of the top contact. Both contacts are oxide-free, due to the electrochemical reduction at the Te interfaces during the in-situ electrodeposition.

For the second set of samples, the contact is performed in a second step. At the end of the CVD growth, MWCNT or Si NW are emerging from the pores at the top of the membrane, over a length of a couple of $100 \mathrm{~nm}$. The density of CNT or NW emerging out of the top of the membrane is very low and not uniform. This is due to the presence of an oxide layer at the interface between the pore and the non-oxidized Al. In the electrolytic bath (during the step before CVD growth), the catalyst nucleates from the Al layer on the bottom of the wire, after the application of the potential. The catalyst is consequently deposited in the wires that have no, or negligibly small, oxide layer. According to SEM observations, some islands of a couple of nanowires are emerging out of the membrane, from part to part, separated by a few microns. It is consequently easy to contact the emerging part of the CNT or NW with a layer of Au, or any other metal (Cu, Co, Ni and Al), through a mask, by evaporation or sputtering. Among all wires or tubes that can be contacted in parallel during the process, we observe (see Section IV) that only a couples of wires with good ohmic contacts effectively contribute to the signal.

\section{COULOMB BLOCKADE REGIME}

The dynamic conductance measurements $G(I)=d I /\left.d V\right|_{I}$ were performed with a lock-in detection bridge LR7OO (using an AC current from $0.3 \mathrm{nA}$ of amplitude for most samples to $10 \mathrm{nA}$ for low resistance samples), together with a DC current $I$ provided by a current source. 
(d)

(a) $20-50 \mathrm{~nm}$

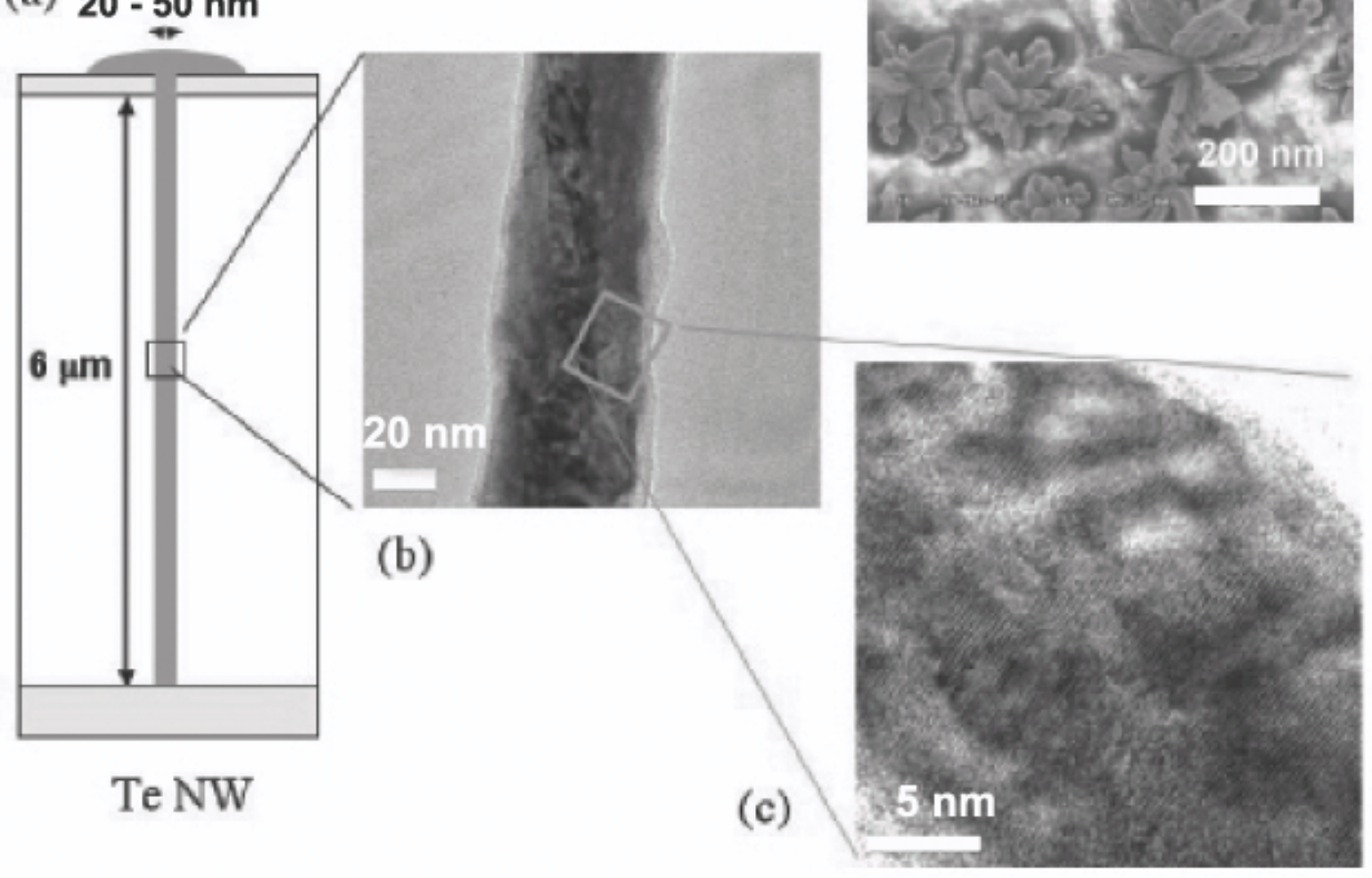

FIG. 1: (a) Sketch of a Te nanowire inside the polycarbonate nanoporous membrane. (b) Transmission microscopy of the wire. (c) High resolution transmission microscopy. (d) View (SEM) of the top of the membrane with the typical dentritic surface growth above the wires.

The curves $G(V)$ plotted as a function of bias voltage are obtained after calculating the voltage $V=\int_{0}^{I}(G(i))^{-1} d i$. The static conductance $G_{D C}(V)$ as a function of voltage is also measured directly with a nanovoltmeter in order to identify possible frequency dependent responses. The profiles $\mathrm{G}(\mathrm{V}, \mathrm{T})$ are measured with temperatures ranging from $T=300 \mathrm{~K}$ to $T=4.2 \mathrm{~K}$. The results, plotted in Fig. 3, show the typical profiles of the G(V) curves at different temperatures, in a standard semi-log plot Fig. 3 (a). The profile shows two remarkable asymptotic regimes: the nearly zero bias regime $e V \ll k T$ ( $k$ is the Boltzmann constant, and $e$ the charge of the electron), where the conductance is a power law of the temperature (Fig. 3 (b)) : 


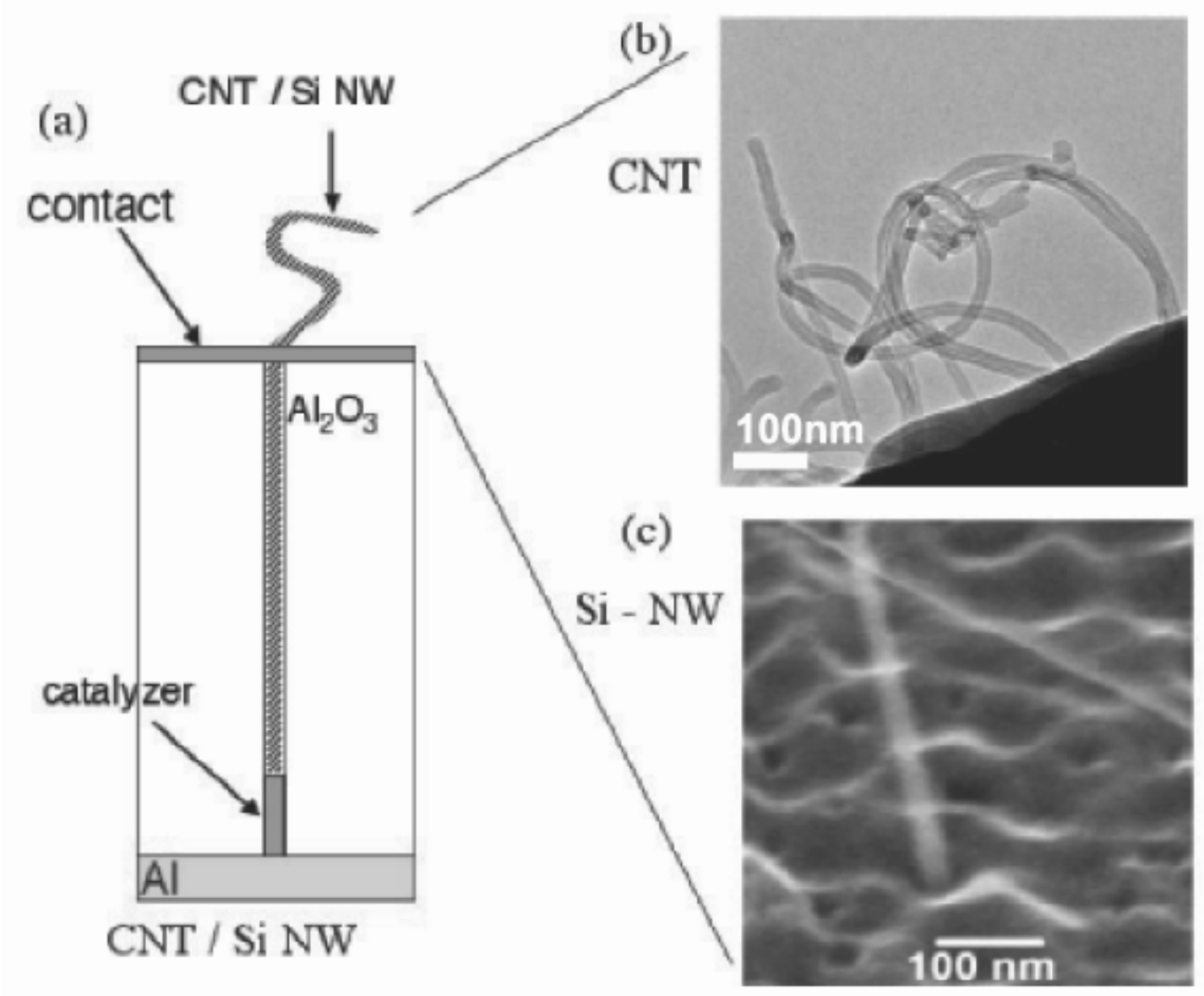

FIG. 2: (a) Sketch of a CNT or Si nanowire inside the alumina nanoporous membrane. Lateral view of the top of the membrane by transmission microscopy of (b) CNT, (c) Si nanowire emerging from membrane.
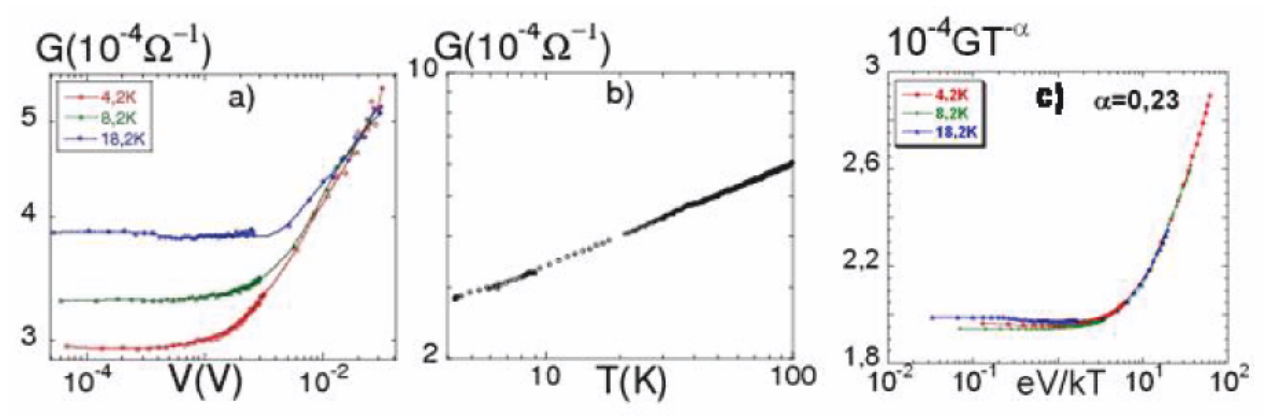

FIG. 3: Typical transport properties of the samples studied (here, of a MWCNT): a) Conductance as a function of bias voltage at different temperatures, b) Zero bias conductance as a function of the temperature, $\mathrm{c})$ Scaling plot $G T^{-\alpha}(\mathrm{eV} / \mathrm{kT})$. 


$$
\lim _{e V \ll k T} G(T)=G_{T} \cdot T^{\alpha}
$$

and the high bias regime $e V \gg k T$, where the conductance is also a power law, but a power law of the bias voltage:

$$
\lim _{e V \gg k T} G(V)=G_{V} \cdot V^{\alpha}
$$

What is significant here is that the power coefficient $\alpha$ is the same in both regimes. The scaling law is valid for all intermediate regimes (Fig. $3(\mathrm{c})$ ): introducing the scaling function $f$, all points collapse on a unique curve

$$
G(T, V) \cdot T^{-\alpha}=f(e V / k T)
$$

This scaling law is presented in Fig. 4 for different samples (CNT, Te NW, and Si NW). The profile, described by the function $f$, is typical and systematically measured in such samples: this is a robust structure that is obtained whatever the type of the wire (carbon nanotubes, Te and Si nanowires, and metallic wires), the nature and crystallinity of the contacts (Co, Ni, Cu, Au, Te,..) [22], the length (300 to $6000 \mathrm{~nm}$ ), the diameter of the wires (from $5 \mathrm{~nm}$ to $200 \mathrm{~nm}$ ), and the nature of the supporting template (polycarbonate and alumina) [23]. Note that a deviation from the scaling law can be observed for the Si nanowires. The origin of this deviation deserves further investigations, and may be related to the crystalline properties of the Si nanowires [46], [47].
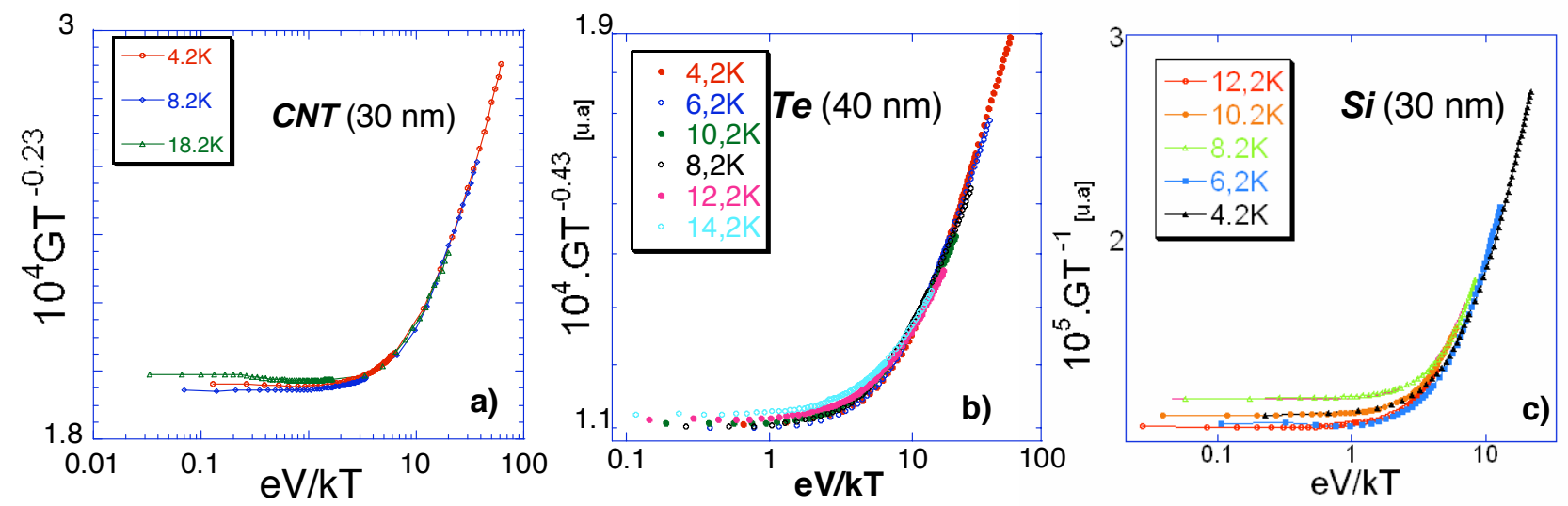

FIG. 4: Examples of scaling laws $G T^{-\alpha}=f(e V / k T)$ observed for: a) a MWCNT of diameter $\mathrm{d}=30 \mathrm{~nm}, \mathrm{~b})$ a TeNW of diameter $\mathrm{d}=40 \mathrm{~nm}$, and c) a SiNW of diameter $\mathrm{d}=30 \mathrm{~nm}$. 
The histogram of the coefficient $\alpha$ obtained for all samples measured is presented in Fig. 5. The fluctuations for fixed experimental parameters (e.g. CNT contacted with Ni, or CNT contacted with $\mathrm{Co}$ ), are of the same order of magnitude as that of the total fluctuations for all materials (the individual distributions can hardly be distinguished). Accordingly, the parameter $\alpha$ is not directly defined by the intrinsic parameters, like the band structure or the density of states. This observation confirms the assumption that $\alpha$ is a measure of disorder. The values found for $\alpha$ are in agreement with that found in the literature for CNT measured with four terminal contacts $[4,16,18,19,22,23,48-55]$.

It is possible to go further in this analysis by correlating the main parameters of this study with the coefficient $\alpha$. The parameter $G_{T}$ (see Eq. (1)) is the conductance extrapolated (or measured) at $\mathrm{T}=1 \mathrm{~K}$ at zero bias. The result is presented in Fig. 6. All points approximately align in a $\log -\log$ plot : beyond the singularity of each samples, a universal behaviour is obeyed by all samples as a function of the scaling parameter $\alpha$.

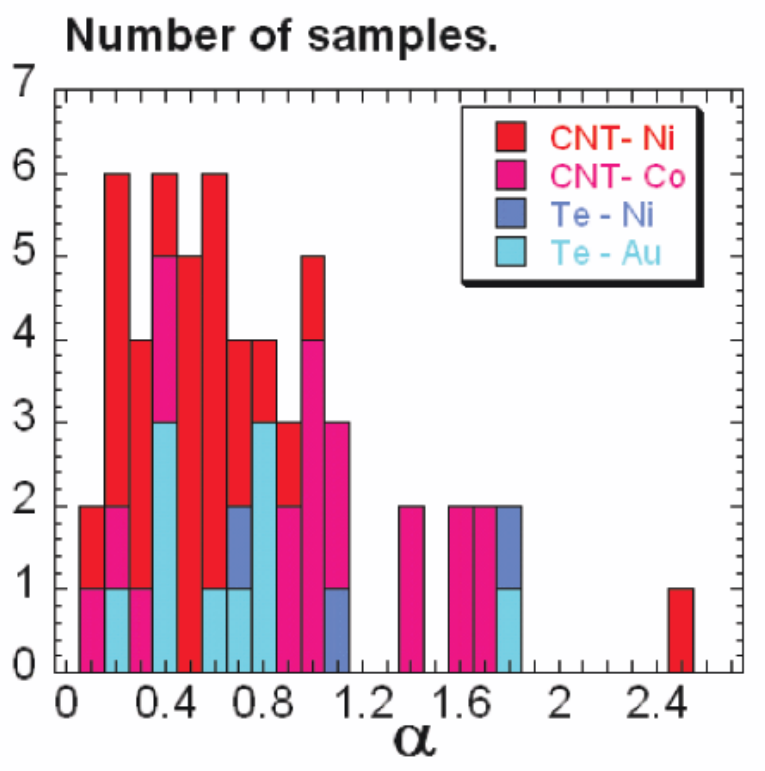

FIG. 5: Histogram of coefficients $\alpha$ obtained for Te NW and CNT samples measured. The parameter $\alpha$ is not directly defined by the intrinsic parameters of the samples.

The law followed by the experimental points plotted as a function of $\alpha$ can be fitted with a formula calculated in the framework of the Coulomb Blockade theories of a single tunnel barrier. In this model, by assuming a well defined tunnel junction, contacted to the rest of the circuit (i.e. mainly the nanowire or nanotube), the following expression is obtained 
$[42,44,45]:$

$$
G_{T} \approx G_{V}(k / e)^{\alpha}=\frac{1}{R} \frac{e^{-\gamma \alpha}}{\Gamma(2+\alpha)}\left(\frac{\pi \alpha k}{e V_{0}}\right)^{\alpha}
$$

where $\gamma=0.577 \ldots$ is the Euler constant and $\Gamma$ the Gamma function. The formula contains two adjustable parameters that are the resistance of the tunnel barrier $R$, and the energy $e V_{0}$ of the junction is the Coulomb energy (e.g. in the case of ultra-small tunnel junctions: $e V_{0}=$ $e^{2} / 2 C$ where $C$ is the capacitance of the tunnel barrier) or the thermal energy $e V_{0}=k T$. This formula is obtained identically for single electron tunnelling for ultra small junctions [42], for extended junctions [43], or in the context of non perturbative dissipative tunnelling (environmental Coulomb blockade) [44, 45]. The fit gives a "universal" resistance of the tunnel junctions of the order of $R=4 k \Omega$, and a "universal" capacitance of the order of $C=10^{-18} \mathrm{~F}$.

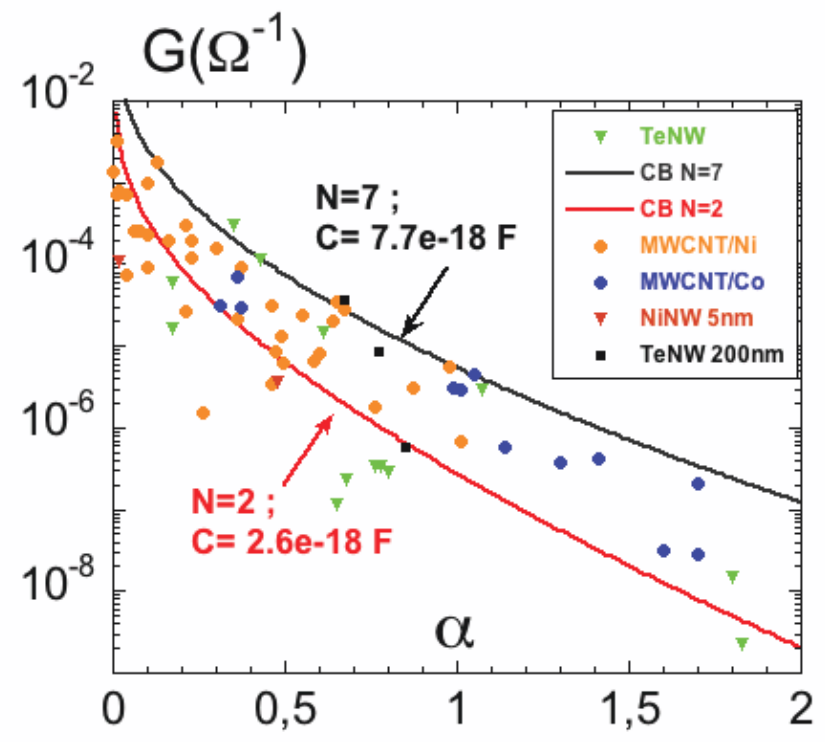

FIG. 6: Prefactor of the conductance $G_{T}(\alpha)$ for about 100 different samples :CNT and Te NW with different contacts (Co and Ni). Lines: fit to Eq. (5) with 2 and 7 junctions.

However, this oversimplified picture of a single junction contacted to the rest of a circuit can hardly be accepted in our experimental study. In the case of a fixed material, e.g. CNTs, we would even expect the opposite situation: a fixed circuit impedance and a distribution of the parameters of the tunnel junction due to different contacts because we changed the 
nature and the crystallinity of the contacts (from the single crystalline $\mathrm{Co}, \mathrm{Cu}$, or $\mathrm{Au}$, to the quasi-amorphous $\mathrm{Ni}$ ) studied in ref [22]. The chemical disorder of the junction is also varying dramatically between electrodeposited Te, in which the contact is performed inside the electrolytic bath under a reducing potential, so is consequently free of oxides, and the CNT or Si nanowires, for which the contact is performed in a second step after the CVD growth, with sputtering, evaporation, or electrodeposition of metal. A universal tunnel junction (with $R=4 k \Omega$ and $C=10^{-18} F$ ) for all these cases is difficult to understand.

Another picture, developed in detail in the last section, can be proposed in terms of a chain of quantum dots (like that observed in references [5]). In this model, the resistance of the junction is not an adjustable parameter but is contained in the variable $\alpha$. The averaged capacitance $C$ of the junctions between the quantum dots is still an adjustable parameter.

The following formula is obtained from simple assumptions developed in the last section of the paper :

$$
\begin{gathered}
G_{T} \approx \frac{e^{2}}{\pi \hbar N \alpha} e^{-(1+\gamma) \alpha}\left(\frac{\pi^{2} \alpha k_{B}}{E_{C}}\right)^{\alpha}, \\
G_{V} \approx \frac{e^{2}}{\pi \hbar N \alpha}\left(\frac{\pi \alpha e}{2 E_{C}}\right)^{\alpha} .
\end{gathered}
$$

with the charging energy $E_{C}=e^{2} / 2 C$, and $N$ referring to the number of tunnel barriers (cf. last section for details).

The corresponding fit is presented in Fig. 6 for two values of the capacitance $C$, which delimited the best fits obtained for all samples. Once $C$ is fixed, the only adjustable parameter is the number $\mathrm{N}$ of tunnel junctions. In this framework, the experimental universal law observed is interpreted as the behavior of arrays of 2 to 7 tunnel barriers of average capacitance between 3 to $8 \mathrm{aF}$ (depending on the sample).

\section{MAGNETORESISTANCE}

The conductance is also measured as a function of a perpendicular magnetic field $H$ applied by a superconducting coil. The field is ramped between $\pm 1.3 T$. MWCNT and TeNW systematically feature a positive magnetoconductance (MC) which (in magnetic field 
and temperature) is in agreement with the formulas of quasi 1D weak localization [23, 25, 26] for diameters below $200 \mathrm{~nm}$. The Si nanowires show a smaller WL effect, or no WL at all (the specificity of the Si nanowires will be reported elsewhere). WL appears in a disordered metallic system, into which electrons undergo Brownian motion due to elastic collisions with defects within a loop of size smaller than the phase coherence length $l_{\phi}$. Weak localisation comes from quantum phase interference effects of electron backscattering [38, 40]. Because the interference terms cancel when applying a sufficiently strong magnetic field (which breaks the time reversal symmetry), WL manifests itself as a positive magnetoconductance.
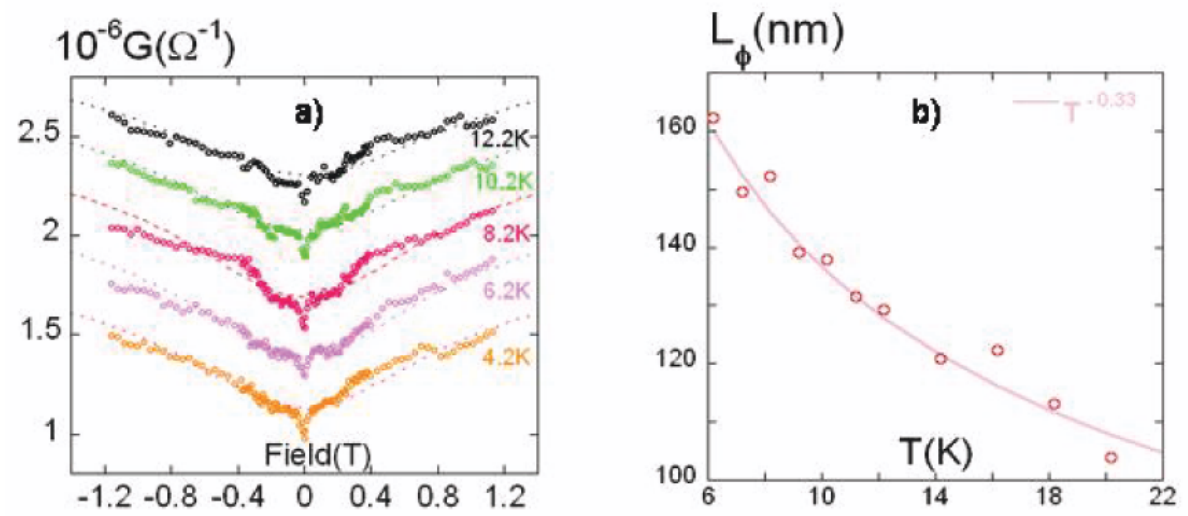

FIG. 7: a) Magnetoconductance of a Te nanowire at several temperatures. Dotted lines: fit to Eq. (7) with parameter $l_{\phi}$ b) Phase coherence length $l_{\phi}(T)$ as a function of the temperature. Grey line: Fit to Eq. (8).

Our samples have magnetoconductive behaviour shown on Fig. 7.a at several temperatures. The variation due to the magnetic field is measured by the quantity $G^{W L}=$ $G(\infty)-G(H)$, where $G(\infty)$ is the saturation value of the conductance, i.e. at a field for which the weak localization correction is destroyed. The doted lines of Fig. 7.a represent the fit to the following quasi 1D WL expression [38, 40]:

$$
G^{W L}=-\frac{1}{\pi} \cdot \frac{e^{2}}{L \hbar}\left(\frac{1}{l_{\phi}^{2}}+\frac{W^{2}}{3 . l_{H}^{4}}\right)^{-1 / 2}
$$

where $l_{H}=(\hbar /(e H))^{1 / 2}$ is the magnetic length $(H$ the magnetic field), $W$ diameter of the wire, $e$ the charge of an electron and $\hbar$ the Planck constant. The adjustable parameters are $G(\infty)$ and $l_{\phi}$. At each temperature, the phase coherence length $l_{\phi}$ can be extracted (cf. Fig.7.b). The function $l_{\phi}(T)$ follows the Altshuler-Aronov-Khmelnitskii [38, 40] formula: 


$$
l_{\phi}(T)=\sqrt{2}\left(\frac{D G_{D} L \hbar^{2}}{e^{2} k T}\right)^{1 / 3}
$$

where $L$ is the length of the sample, $k$ the Boltzmann constant, $D$ the diffusion coefficient, and $G_{D}$ the conductivity without WL correction (i.e. the Drude conductivity).

Hence, we can associate with each sample a couple of transport parameters $\left(\alpha, l_{\phi}(T=\right.$ $4.2 K)$ ). By representing these couple of parameters for all the samples studied we observed a strong correlation between $\alpha$ and $l_{\phi}$ : the scaling exponent $\alpha$ is decreasing as a function of the phase coherence length. This curve can be reproduced at different temperatures, and also presents a universal behavior. The correlation at $\mathrm{T}=4.2 \mathrm{~K}$ is shown in Fig. 8. The data fits a power law of power coefficient :

$$
\alpha\left(l_{\phi}\right)=c . l_{\phi}^{-3 / 2}
$$

where the adjustable parameter $c$ depends on the temperature. A derivation of this empirical law is reported in Sec. VI, for the model of the chain of quantum dots of typical size below $l_{\phi}$. Unfortunately, the adjustable parameter $c$ does not give direct physical information on the system due to numerical constants difficult to express precisely (see the discussion of Eq. (15) in section VI).

It is worth pointing out that, in contrast with usual systems exhibiting WL (e.g. in metallic samples), the conductivity without WL (at saturating magnetic field) is not the usual Drude conductivity but the Coulomb blockade regime described in the previous section. The observation of the WL and the description with Eqs. (7) and(8) is consequently not trivial. As an illustration, Fig. 9 shows that the two effects, WL and Coulomb blockade, are not independent. The conductance $G(V, T ; H)$, with the typical zero-bias anomaly profile, is plotted at different magnetic fields $H$ : it can be observed that bias voltage destroys the weak localization correction. The curves are plotted for the two temperatures, $4.2 \mathrm{~K}$ and $8 \mathrm{~K}$, in order to show that the destruction is not produced indirectly by the Joule heating (otherwise, the profiles at $8 \mathrm{~K}$ would scale to those at $4.2 \mathrm{~K}$ at lower bias).

\section{LOW TEMPERATURE TRANSITION}

The low temperature measurements are made in a dilution refrigerator down to $50 \mathrm{mK}$. Resistance is measured by traditional two contact lock-in detection, with AC excitation be- 


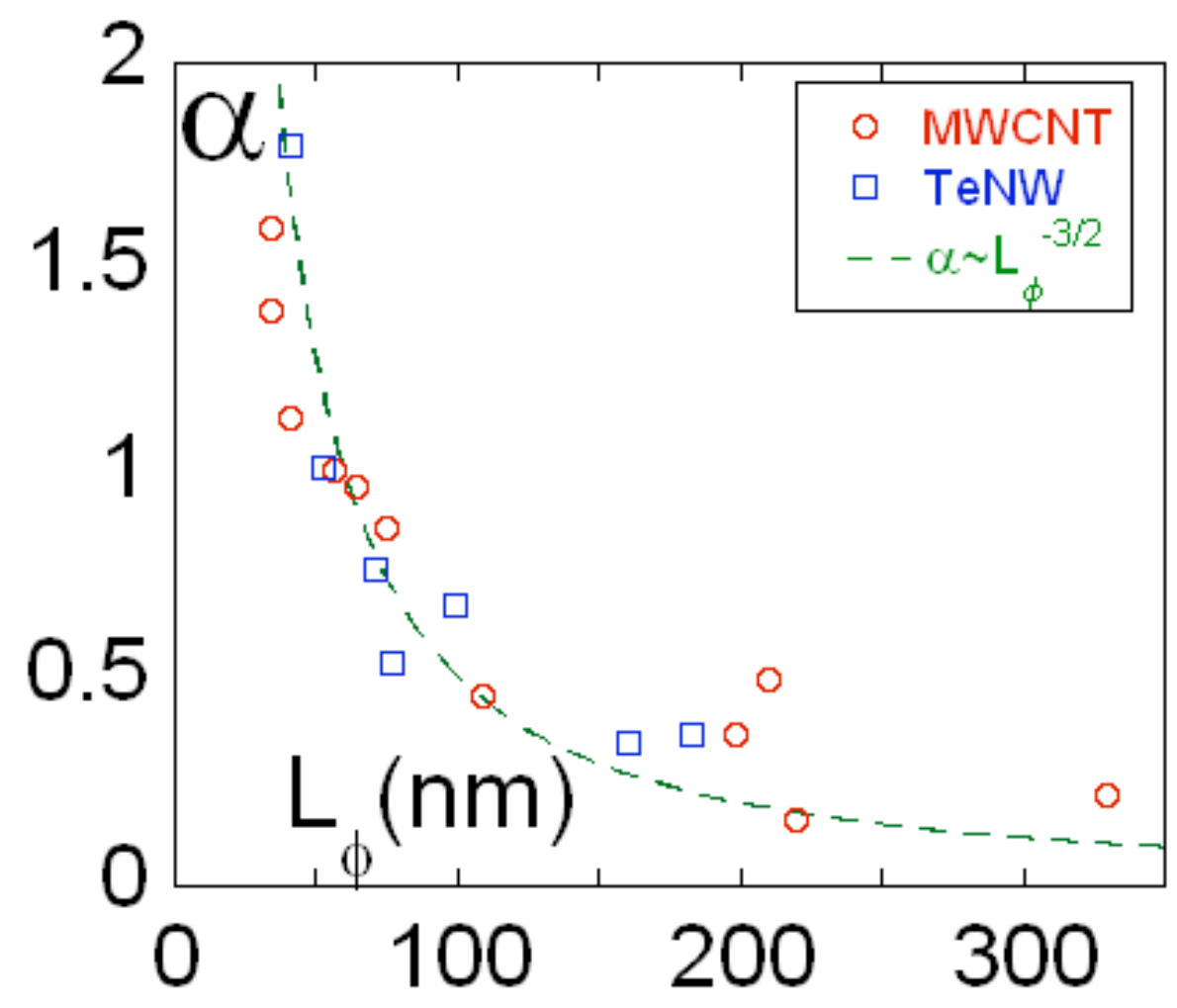

FIG. 8: Correlation between the phase coherence length $l_{\phi}$ at $4.2 \mathrm{~K}$ and $\alpha$ for the 20 samples studied. The dotted line is the Fit defined by Eq. (9)

tween 10 and $100 \mu \mathrm{V}$. A DC bias is applied in parallel to probe the bias voltage dependence of the resistance. The response of the samples is measured by the lock-in after having been amplified by a $10^{8}$ current amplifier, which allowed us to probe resistance up to the $\mathrm{G} \Omega$ range.

\begin{tabular}{|c|c|c|c|c|c|c|c|}
\hline Name & material & $\mathrm{L}(\mu \mathrm{m})$ & $\mathrm{d}(\mathrm{nm})$ & $\alpha$ & $E_{a}(\mathrm{meV})$ & $E_{C}(m e V)$ & $T_{a}(K)$ \\
\hline A & MWCNT & 1 & 30 & 2.5 & 0,81 & 7.25 & $7-8$ \\
\hline B & MWCNT & 1 & 30 & 2 & 0,82 & 5 & $5-6$ \\
\hline C & MWCNT & 1 & 30 & 0.85 & 0,08 & 0.48 & $0,2-0,3$ \\
\hline D & MWCNT & 1 & 30 & 1.2 & 0,45 & 3 & $4-5$ \\
\hline E & TeNW & 6 & 150 & 0.17 & 0,04 & 0.18 & $6-7$ \\
\hline F & TeNW & 6 & 40 & 1.1 & 0,22 & 1 & $4-5$ \\
\hline
\end{tabular}

TABLE I: Sample description: Name, material, length $\mathrm{L}(\mu \mathrm{m})$, diameter $\mathrm{d}(\mathrm{nm})$, scaling exponent $\alpha$, activation energy $E_{a}$, critical energy $E_{C}=e V_{C}$, and transition temperature $T_{a}$. 


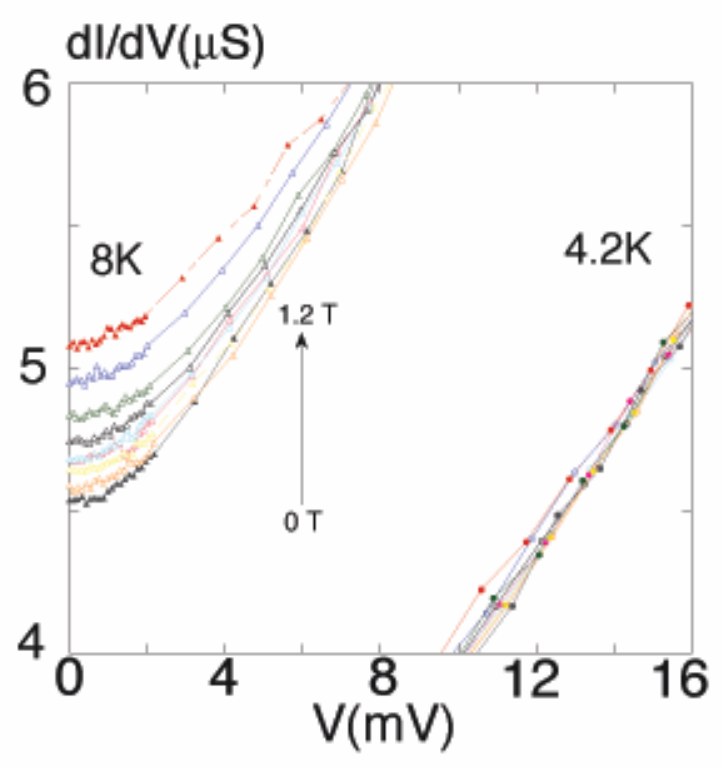

FIG. 9: Zoom of the conductance as a function of bias voltage for different magnetic fields (0T to $1.2 \mathrm{~T}$ ), at $4.2 \mathrm{~K}$ and $8 \mathrm{~K}$.

We studied the transport behavior of 6 samples at very low temperature (cf. Table 1) : 4 MWCNT (samples A-D) of $1 \mu \mathrm{m}$ long with a diameter $\mathrm{D}=30 \mathrm{~nm}$, one TeNW (sample E) of $6 \mu \mathrm{m}$ long with a diameter of $150 \mathrm{~nm}$, and one TeNW (sample F) of $6 \mu \mathrm{m}$ long with a diameter around 40nm. For all samples, at zero bias, we observed an abrupt transition in the electronic transport regime at a critical temperature $T_{a}$ (Fig. 10). The transition occurs from the power law regime previously described to an activated regime at $T<T_{a}$ (see inset in Fig. 10). More precisely, the samples A,B,C,D,F showed a transition from the power law $G \propto T^{\alpha}$ to an activated law $G \propto \exp \left(-\frac{E_{a}}{k T}\right)$, with $E_{a}$ designating the activation energy. The parameter $E_{a}$ is also a characteristic of each sample( cf. Fig. 10.(a-e)). Sample E (whose diameter is 5 times bigger than the other samples) shows a behavior which cannot be fitted to a simple activation law (cf. Fig.11.a), but has rather a profile of the form $G \propto \exp \left(-\sqrt{\frac{E_{a}}{k T}}\right)$ (see Figure.11). We will come back to the reason for this difference below. The results are summarized in table I.

The behavior of the conductance as a function of the bias voltage at low temperature is shown in Fig. 12 in a semi-log plot for samples A to F. At $T>T_{a}$, the conductance follows the scaling law reported in the previous sections. For $T \leq T_{a}$, we systematically observed a critical voltage $V_{C}$ below which the samples change from the scaling law regime to an 

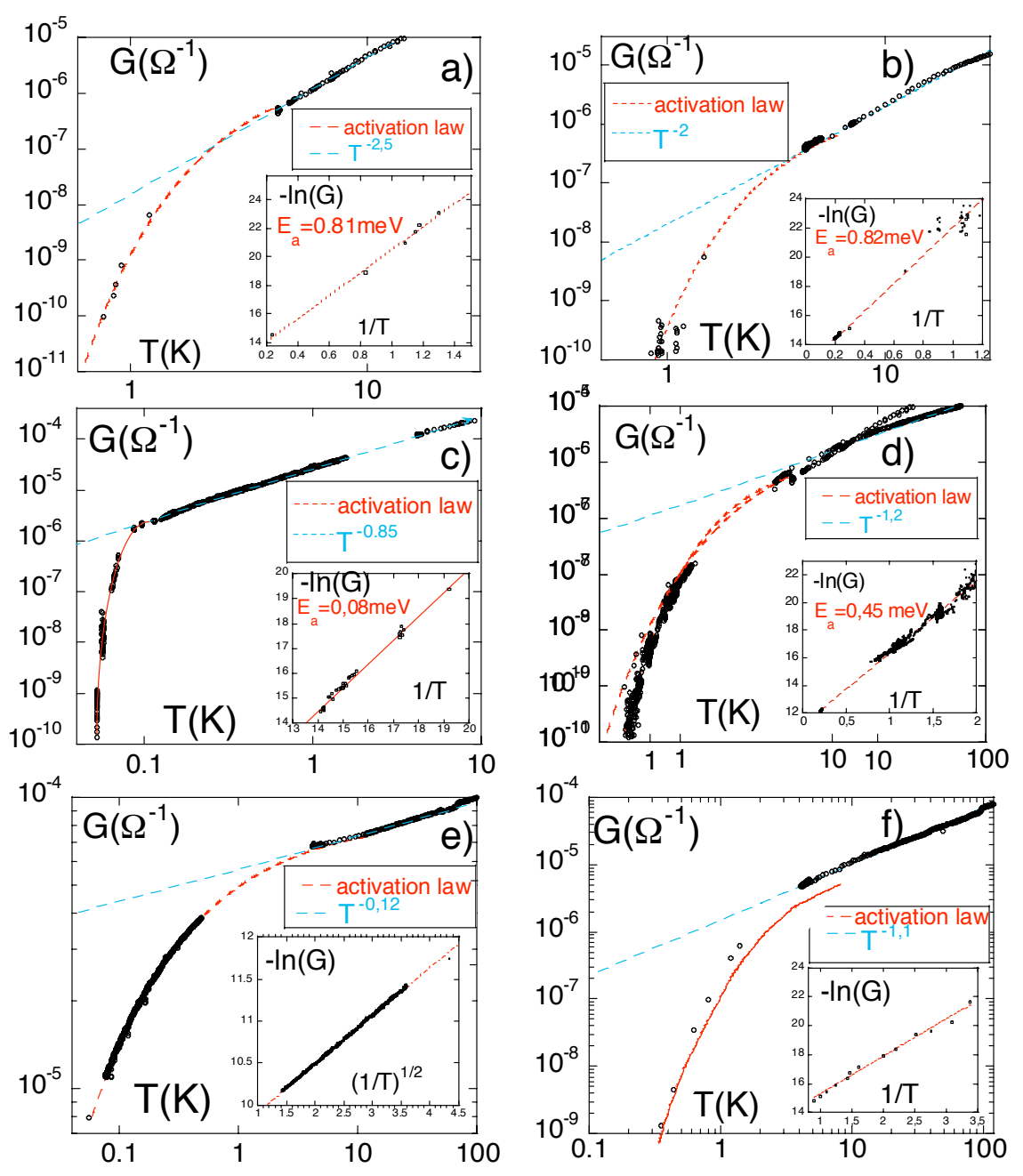

FIG. 10: Conductance as a function of temperature at zero bias voltage for six samples: (a-d) 4 MWCNT (samples A,B,C, and D), (e): TeNW of 150nm diameter (sample E), and TeNW of $40 \mathrm{~nm}$ diameter (sample F). Dotted line: power law of the Coulomb blockade regime. Inset: Arrhenius plot at law temperatures $\left(T \leq T_{a}\right)$.

exponential dependence:

$$
G \propto \exp (-C(T) \cdot V)
$$

where $C(T)$ is a function of the temperature.

The transition can be very sharp, with a jump of at least three orders of magnitude of the resistance (cf. Fig. 12.b and Fig. 12.d). The plateau observed for the conductance $G=10^{-9} \Omega^{-1}$ is an artifact due to the limitation of the measurement set-up. It is important 


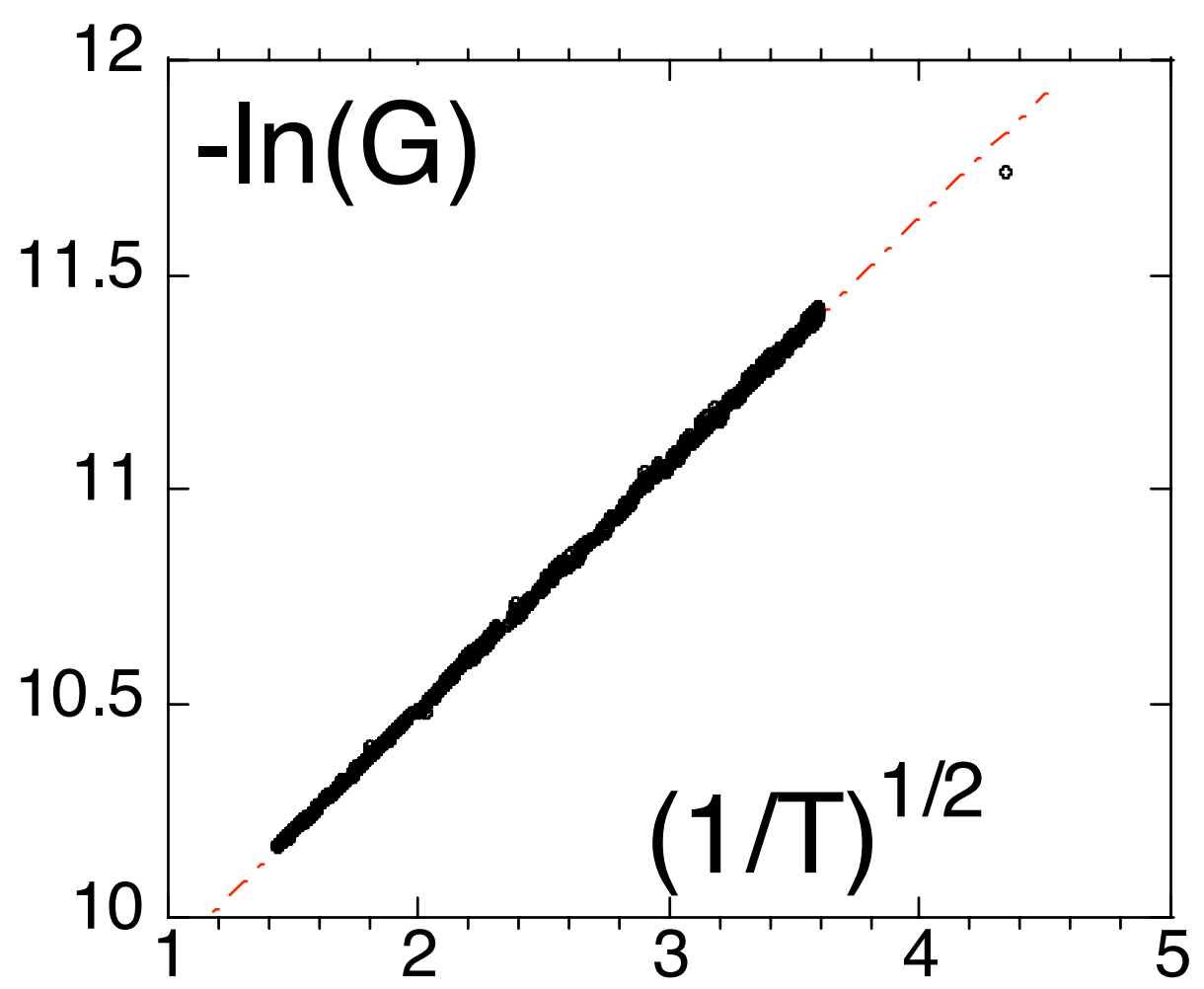

FIG. 11: Arrhenius-like Plot of the conductance of sample E (bigger diameter: $150 \mathrm{~nm}$ ) at zero DC bias and below $T_{a}$. Dotted line: fit with the law $G \propto \exp \left(-\sqrt{\frac{E_{a}}{k T}}\right)$.

to note that the transition is not that observed for metal - insulator transition [56], because it occurs from a Coulomb blockade regime to the insulator state, as shown in the scaling plot Fig. 13. Whatever the temperature, the Coulomb blockade regime, with the correct scaling coefficient $\alpha$ is recovered below $T_{a}$, under a high enough bias voltage. In other terms, the scaling law is maintained whatever the temperature, for high enough scaling parameter $e V / k T$.

Once again, in the activation regime, a strong correlation appears when we measure a couples of parameters $\left(\alpha, E_{a}\right)$ for all samples studied (Fig. 13) $: E_{a}$ follows an increasing function of $\alpha$ (Fig. 14). The same trend is observed for $E_{C}=e V_{C}$ plotted as a function of $\alpha$ (not shown). An interpretation of this third universal law is provided by the model of a chain of quantum dots of high conductance developed in the last part of this article (see section VI). In the framework of this model, it appears that there exists a critical temperature $T_{a}$ at which the conductance goes to zero. The activation energy is related to 

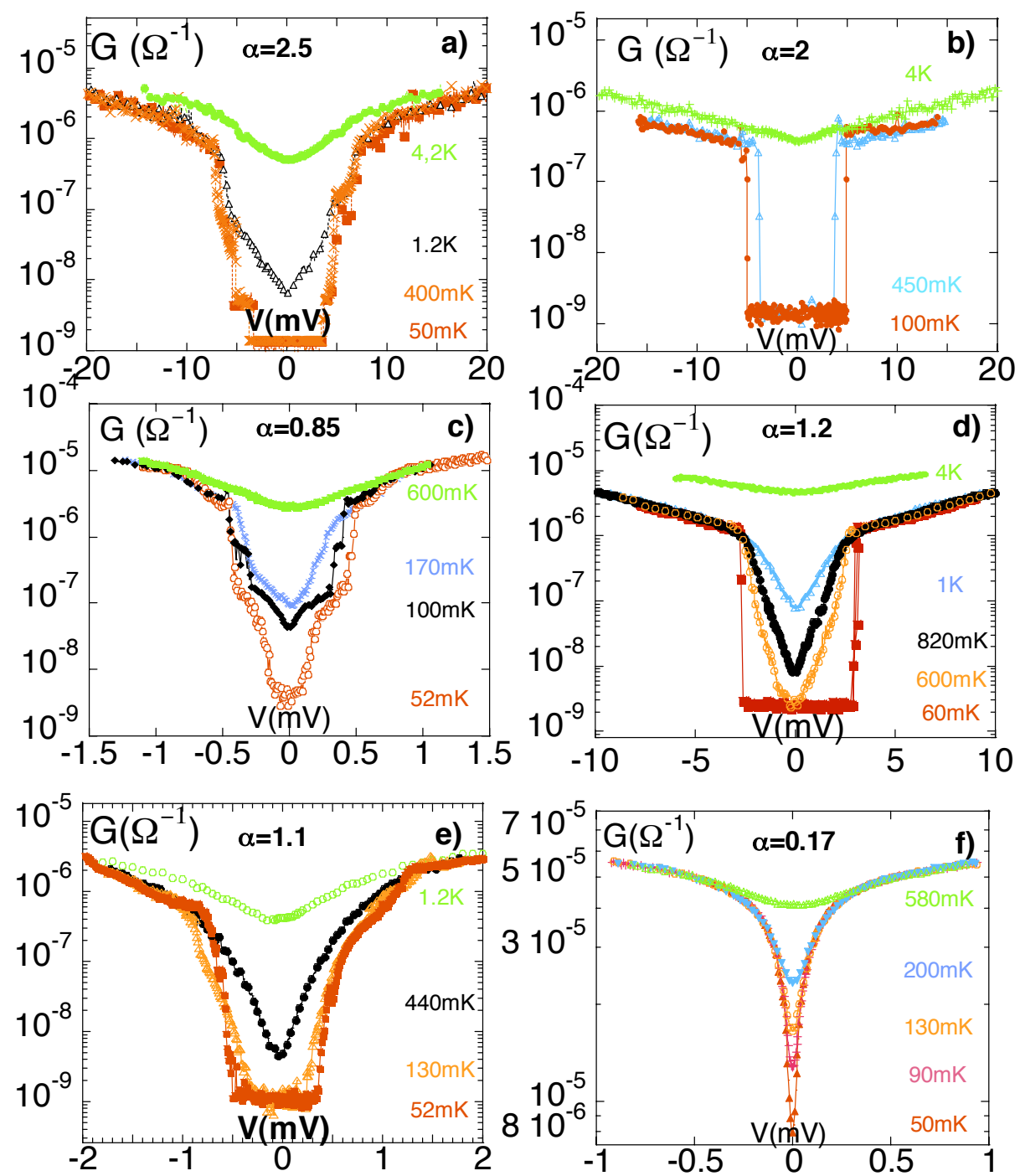

FIG. 12: Conductance versus DC bias voltage for each of the sample A to F at different temperature below $T_{a}$, in a semi-log scale. We observe for each sample a strong increase of the resistance bellow a critical voltage $V_{C}$.

the temperature $T_{a}$ through the activation law: critical energy $E_{a}=k_{B} T_{a} \ln \left(\tau / \tau_{0}\right)$ where $\tau$ is the typical measurement time and $\tau_{0}$ is the typical electronic relaxation time). Each tunnelling junction (of resistance $\mathrm{R}$ ) enters into an activated behavior [57, 58], and gives to the sample conductance an averaged activation process. In order to fit the data, the following expression is used (see discussion in section VI Eq.(25) and Eq.(27)):

$$
E_{a} \approx \frac{N-1}{\alpha} \exp \left(-\frac{N-1}{\alpha}\right)
$$



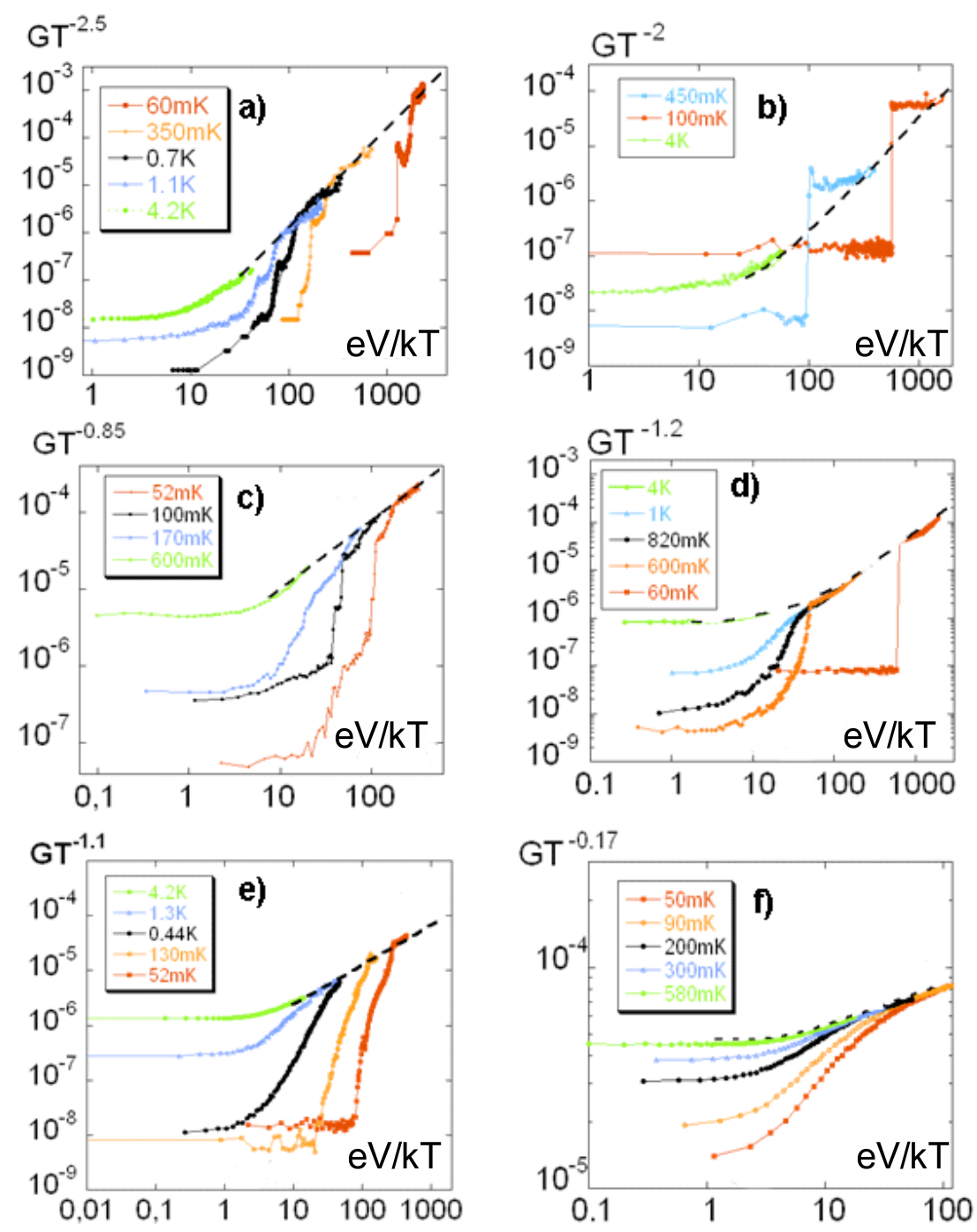

FIG. 13: Scaling plot of the function $G T^{-\alpha}$ as a function of the ratio $e V / k T$ (same data as Fig.12).

Dotted line: scaling law

The best fit is obtained for an average number of 5 junctions (dotted line of Fig. 14)

The transition observed from the power law regime to the activated one (cf. Fig. 13) could be explained by the transition to a strong tunnelling regime of the most resistive junctions. In this framework, the voltage behavior of the resistance below $T_{a}$ can also be understood. When a DC bias is applied, the barrier level of each junction is decreased. Once a critical value $V_{C}$ is reached, the sample comes back to the scaling law regime. Another estimation of the number of barriers existing in our sample can be obtained by the ratio of the critical energy $E_{C}$ to the activation energy $E_{a}$ which, depending on the sample, takes 
the value $E_{C} / E_{a} \approx 5-9$. These values are in good agreement with those deduced from the universal law of Fig. 6. The correlations observed between $E_{a}$ and $\alpha$ can be fitted to Eq.11 quite well, and give a mean value of the number of junctions around 5, which is in agreement with the previous values.

Sample E is different, because of its 3 times bigger diameter $(150 \mathrm{~nm})$. As the diameter of the wire increases, the equivalent behavior goes from a 1-dimensional chain of tunnel junctions to a 3-dimensional one. Then, many chains in parallel appear, and electrons have many paths to go through the wire. Therefore, the final resistance results from adding up several activation exponents with distributed charging energies, and results in the $R \approx \exp \left(-\sqrt{\left(E_{a} / k T\right)}\right)$ law observed.

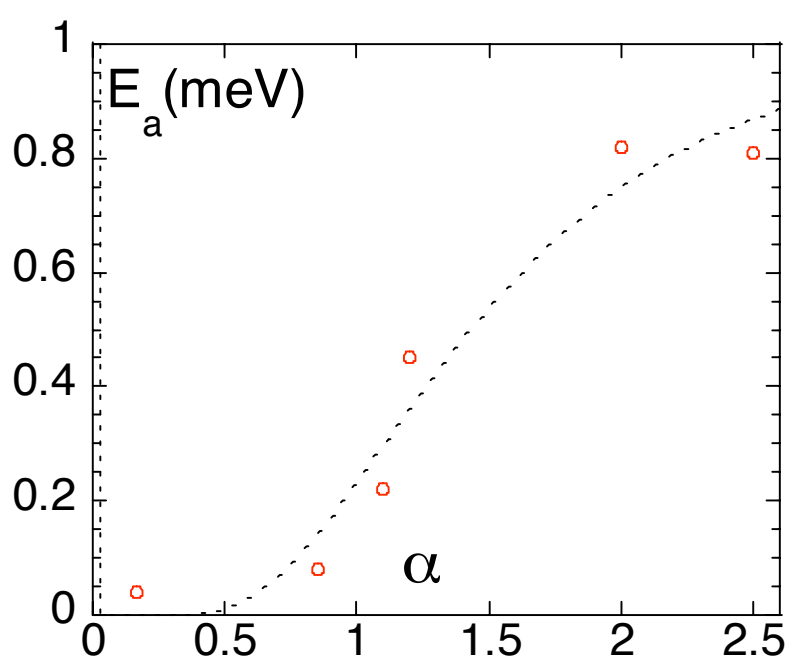

FIG. 14: Activation energy $E_{a}(\alpha)$ as a function of the scaling coefficient $\alpha$ for 6 samples. Dotted line: fit with Eq. (11) (see text).

\section{THEORETICAL DESCRIPTION OF THE QUANTUM DOT ARRAY MODEL.}

In this section we develop a theoretical model, which has been used to fit the experimental data. We consider a homogeneous one-dimensional chain of $N-1$ macroscopically identical quantum dots.

Let us first briefly discuss the main features of this model and its relation to the ex- 
periment. First of all, the experimentally observed suppression of the conductance with decreasing temperature and bias voltage is attributed to Coulomb blockade in the tunnel junctions. This assumption allows us to reproduce the main features observed in the experiment. Indeed, at sufficiently high temperatures and at low resistance of a tunnel junction, $R \ll 2 \pi \hbar / e^{2}$, the regime of weak Coulomb blockade is realized, in which the model predicts logarithmic dependence of the conductance on temperature. The latter is similar to the power law dependence observed in the experiment. At the same time at temperatures lower than certain effective charging energies the strong exponential suppression of the conductance is expected, which is again in line with experimental observations. Next, both in theory and in experiment the non-linear differential conductance shows similar behavior as a function of bias voltage. Finally, in order to describe the magnetic field dependence of the conductance we include the weak localization correction.

We now turn to the quantitative analysis of the proposed model. The quantum dots are coupled to each other and to the leads by $N$ identical tunnel junctions with resistance $R$. We also introduce a capacitance $C$ between neighbouring quantum dots. Provided $R$ is sufficiently low, namely $R \ll 2 \pi \hbar / e^{2}$, the differential conductance of this system is given by the sum of three contributions

$$
\frac{d I}{d V}=\frac{1}{N R}+G^{\mathrm{WL}}+\frac{d I^{\mathrm{CB}}}{d V} .
$$

The first and leading term in this expression, $1 / N R$, comes from the classical addition of junction resistances. The second term, $G^{\mathrm{WL}}$, is the weak localization correction to the conductance, while the last term, $d I^{\mathrm{CB}} / d V$, is the interaction correction to the current originating from Coulomb blockade effects. Both $G^{W L}$ and $d I^{\mathrm{CB}} / d V$ are proportional to $e^{2} R / 2 \pi \hbar \ll 1$ and formally supposed to be small compared to the leading classical contribution:

Let us first consider the weak localization correction to the conductance $G^{\mathrm{WL}}$. Provided the dephasing length $l_{\phi}$ exceeds the size of a single quantum dot and the magnetic field is zero, the weak localization correction reads [59]

$$
G^{\mathrm{WL}}=-\frac{e^{2}}{\pi \hbar N} \frac{l_{\phi}}{d}
$$

where $l_{\phi}$ is the dephasing length and $d$ is the size of a quantum dot. Note that if we introduce the length of the sample $L=N d$, Eq. (13) coincides with the standard result 


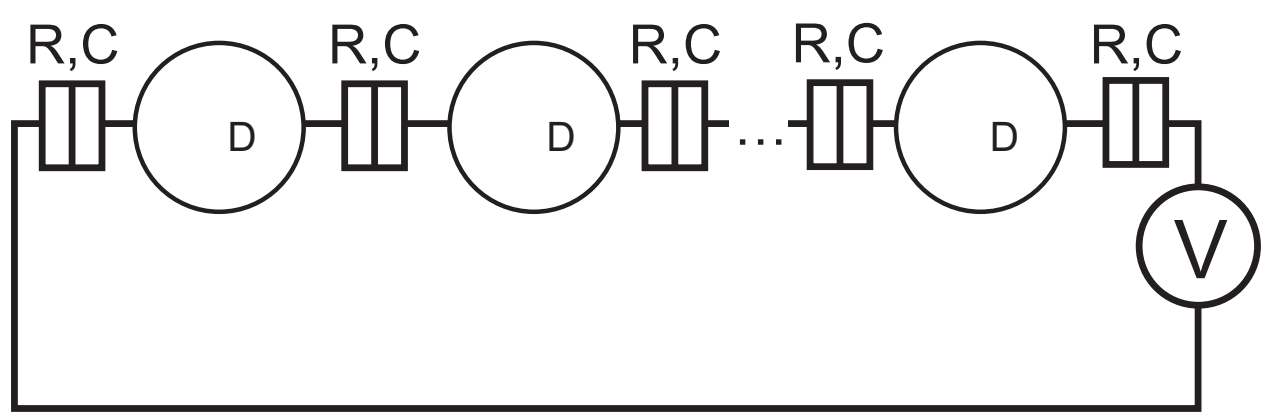

FIG. 15: Model of the chain of quantum dots coupled by tunnel junctions.

derived for diffusive metals (see e.g. Ref. [39]). Since the temperatures in the experiment, $T \gtrsim 1-2 \mathrm{~K}$, are relatively high and exceed the level spacing in a single quantum dot $\delta$, we can use the expression for $l_{\phi}$ derived by Altshuler, Aronov, Khmelnitski [38]

$$
l_{\phi}=a\left(\frac{D G_{D} L \hbar^{2}}{e^{2} k_{B} T}\right)^{1 / 3} .
$$

Here $a$ is a numerical pre-factor. In order to adapt this formula to the array of quantum dots we use the expression for the diffusion constant (see e.g. [40, 59]) defined by the drude conductivity $\sigma \equiv d /(W R)=2 e^{2} D /(W d \delta)$ ( $W$ is the diameter). Besides that, we rewrite the product $G_{D} L$ in the form $G_{D} L=L / N R=d / R$. Thus we obtain

$$
l_{\phi}=a d\left(\frac{\hbar^{2} \delta}{2 e^{4} R^{2} k_{B} T}\right)^{1 / 3}
$$

Next we turn to the interaction correction $d I^{\mathrm{CB}} / d V$. The dwell time is defined by the expression $\tau_{D}=e^{2} R / \delta$. In the diffusive limit $2 \pi k_{B} T \tau_{D} / \hbar, e V \tau_{D} / \hbar \ll 1$ and $N \gg 1$ this correction has the standard $1 / \sqrt{T}, 1 / \sqrt{e V}$ dependence on temperature and voltage [39]. However, in our experiments the opposite limit $2 \pi k_{B} T \tau_{D} / \hbar, e V \tau_{D} / \hbar \gtrsim 1$ is realized. In this case and for $\tau_{D} \gg R C$ the correction $d I^{\mathrm{CB}} / d V$ takes the form $[60]$

$$
\begin{aligned}
\frac{d I^{\mathrm{CB}}}{d V}= & -\frac{e^{2}}{\pi \hbar} \frac{N-1}{N^{2}} \operatorname{Re}\left[\Psi\left(1+\frac{\hbar}{2 \pi k_{B} T R C}+\frac{i e V}{2 \pi k_{B} T}\right)\right. \\
& +\left(\frac{\hbar}{2 \pi k_{B} T R C}+\frac{i e V}{2 \pi k_{B} T}\right) \Psi^{\prime}\left(1+\frac{\hbar}{2 \pi k_{B} T R C}+\frac{i e V}{2 \pi k_{B} T}\right) \\
& \left.-\Psi\left(1+\frac{i e V}{2 \pi k_{B} T}\right)-\frac{i e V}{2 \pi k_{B} T} \Psi^{\prime}\left(1+\frac{i e V}{2 \pi k_{B} T}\right)\right]
\end{aligned}
$$


Where $\Psi(x)=\frac{\Gamma^{\prime}(x)}{\Gamma(x)}$ is the digamma function. In the limit $e V \lesssim 2 \pi k_{B} T \lesssim \hbar / R C \mathrm{Eq}$. (16) reduces to

$$
G(T)=\frac{1}{N R}\left[1+N R G^{\mathrm{WL}}-\frac{N-1}{N} \frac{e^{2} R}{\pi \hbar}\left(\ln \frac{\hbar}{2 \pi k_{B} T R C}+1+\gamma\right)\right]
$$

Likewise for $2 \pi k_{B} T \lesssim e V \lesssim \hbar / R C$ we find

$$
G(V)=\frac{1}{N R}\left[1+N R G^{\mathrm{WL}}+\frac{N-1}{N} \frac{e^{2} R}{\pi \hbar} \ln \frac{e V R C}{\hbar}\right]
$$

As we have discussed earlier, Eqs. $(17,18)$ are the result of the lowest order expansion in the dimensionless parameter $e^{2} R / \pi \hbar \ll 1$. In this limit and at sufficiently high temperatures and voltages $\left(k_{B} T, e V \gtrsim E_{a}\right.$, where $E_{a}$ will be defined below, see Eq. (25)), we may formally replace Eqs. $(17,18)$ by the following ones:

$$
\begin{gathered}
G(T) \approx \frac{e^{2}}{\pi \hbar N \alpha} e^{-(1+\gamma) \alpha}\left(\frac{\pi^{2} \alpha k_{B} T}{E_{C}}\right)^{\alpha}, \\
G(V) \approx \frac{e^{2}}{\pi \hbar N \alpha}\left(\frac{\pi \alpha e V}{2 E_{C}}\right)^{\alpha} .
\end{gathered}
$$

Here we defined the charging energy $E_{C}=e^{2} / 2 C$ and the exponent

$$
\alpha=\frac{(N-1) e^{2} R}{\pi N \hbar\left(1-N R\left|G^{\mathrm{WL}}\right|\right)}
$$

Although the weak localization correction $G^{\mathrm{WL}} \propto T^{-1 / 3}$ depends on temperature, this dependence is weak and in the wide range of temperatures $\alpha$ approximately remains constant. This result justifies the analysis of the data presented in Fig. 6. At the same time $\alpha$ is sensitive to magnetic field through the same weak localization correction $G^{\mathrm{WL}}$. In the metallic limit we have $N R\left|G^{\mathrm{WL}}\right| \ll 1, N \gg 1$ and the exponent $\alpha$ acquires a very simple form

$$
\alpha \approx \frac{e^{2} R}{\pi \hbar}
$$

Let us now express the dephasing length $l_{\phi}(15)$ in terms of $\alpha$. The level spacing in a quantum dot depends on its size as $\delta \sim 1 / N_{0} d^{3}$, where $N_{0}$ is the density of states at the Fermi level. Therefore, 


$$
l_{\phi}=\frac{b}{\alpha^{2 / 3}\left(N_{0} k_{B} T\right)^{1 / 3}},
$$

where $b$ is another numerical prefactor. Here we used the simplified expression for $\alpha$ (22). This result is in agreement with the empirical law Eq. (9) observed in Fig. 8.

Finally, we estimate the temperature $T_{a}$ at which the crossover to the activation behavior should occur. We define $T_{a}$ as the solution of equation $G_{T}\left(T_{a}\right)=0$, where $G_{T}$ is given by Eq. (19). $T_{a}$ sets the scale of temperatures below which the Coulomb blockade correction to the conductance becomes comparable to the linear conductance $1 / N R+G^{\mathrm{WL}}$. In this way we obtain

$$
T_{a} \approx \frac{E_{C}}{\pi^{2} \alpha k_{B}} e^{1+\gamma-1 / \alpha} .
$$

The activation energy $E_{a}$ should be approximately equal to $k_{B} T_{a}$ (assuming that the prefactor $\ln \left(\tau / \tau_{0}\right)=1$ : see last section), i.e. we can put

$$
E_{a}=k_{B} T_{a}=\frac{E_{C}}{\pi^{2} \alpha} e^{1+\gamma-1 / \alpha} .
$$

Likewise we find the critical voltage

$$
V_{C} \approx \frac{2 E_{C}}{\pi \alpha e} e^{-1 / \alpha} .
$$

The activation energy (25) agrees with the estimates of the renormalized charging energy of a tunnel junction obtained in Refs. $[61,62,63]$. Our experimental data rather suggest

$$
E_{a} \sim \frac{e^{-c / \alpha}}{\alpha},
$$

where $c \approx 4$, i.e. the measured activation energy is lower than that predicted by Eq. (25). Two different mechanisms may account for this discrepancy. First, the junctions in the array may have different resistances. In this case the exponent in $E_{a}$ is mostly determined by the junctions with lower resistances, while $\alpha$ is mostly sensitive to high resistances. Therefore the activation energy turns out to be suppressed, $E_{a} \lesssim e^{-1 / \alpha}$. Alternatively, additional suppression of the activation energy may be explained if we assume that the tunnel junctions 
have few channels with significant transmission probability $T_{n} \sim 1$. According to Nazarov [62] in this case one finds the activation energy in the form (27) with $c>1$.

Although Eqs. $(19,20,23,25)$ are formally justified in the limit $\alpha \ll 1$, they seem to describe experimental data quite well even for $\alpha>1$. Since the range $\alpha \sim 1$ is most difficult for theoretical studies, we do not completely understand why it happens. We can speculate that if the junctions in the array have different resistances one can approximately split the system into several parts consisting of one highly resistive junction and several low resistive ones. The low resistive junctions form an Ohmic environment for the poorly conducting one. The conductance of every part is then described by the theory of Coulomb blockade in the presence of electro-magnetic environment [64], which predicts power law dependence $G(T) \sim T^{\alpha}$. The resistance of the whole sample is simply the sum of resistances of the wire sections, and therefore the total conductance $\propto T^{\alpha}$ as well. More accurate approach to this problem should probably rely on the renormalization group equations formulated in Ref. [63].

\section{CONCLUSION}

Transport measurements of an ensemble of semiconductor nanowires and carbon nanotubes have been performed. The samples are obtained by electrodeposition and CVD in nanoporous membranes. Three different kinds of samples have been measured and compared: carbon nanotubes, Te nanowires, and silicon nanowires. Qualitatively, the transport parameters of all samples follow the same profile as a function of bias voltage, temperature, and magnetic field, whatever the material and the contacts. The Coulomb blockade regime dominates in the temperature range between about $50 \mathrm{~K}$ down to about $1 \mathrm{~K}$. The Coulomb blockade regime is characterized by a typical scaling law of the temperature and bias voltage, from which a scaling coefficient $\alpha$ is deduced. The parameter $\alpha$ describes the disorder, and is a fingerprint of each sample. In the Coulomb blockade regime, a weak localization like behaviour is also observed in the magnetoresistance profile. These measurements allow the phase coherence length to be measured as a function of $\alpha$. Beyond, we observe that the weak localization is destroyed at high voltage: an interplay between both effects is evidenced. Finally, below $1 \mathrm{~K}$ (down to $60 \mathrm{mK}$ ), a transition to activation conduction regime is observed at low enough bias potential. The activation energy has been measured as a function of the 
coefficient $\alpha$.

We have observed that the three following parameters : conductance, phase coherence length, and activation energy are universal functions of the coefficient $\alpha$ (in the sense that it is valid for almost all samples measured).

In order to explain the universal laws, a theoretical model of a chain of quantum dots has been developed. This physical picture is supported by recent imaging experiments based on scanning probe techniques showing that nanowires or nanotubes break into a onedimensional chain of quantum dots [3-5] at low temperatures.

Due to charge inhomogeneities, the wire is composed by high conducting regions separated by poorly conducting ones. Schematically, the low conducting regions are associated with tunnel barriers, that separate high conducting regions, quasi-ballistic at this scale, i.e. quantum dots.

The problem is then to understand the consequences of such a picture in terms of transport properties measured in the configuration of two point contacts. The conclusion is that the universal laws associated with the three different regimes are indeed rather well described within this model. The parameter $\alpha$ is defined in relation to the scaling law of the conductance, the phase coherence length, and the activation energy. However, the generalization of the theory to large exponents $\alpha$ is still unclear. Some arguments are proposed in order to relate the model of chain quantum dots to the environmental Coulomb blockade. Another point that remains to be clarified concerns the observed interplay between Coulomb blockade and weak localization for large $\alpha$.

However, this study shows that a change of paradigm should be taken into account while considering the transport properties in carbon nanotubes or semiconductor nanowires. In the context of room temperature nanoelectronic devices, the tubes or wires are usually characterized with the parameters related to bulk semiconductor physics like band structure and mobility of charge carriers. At low temperature, the process of "breaking the wire into a chain of quantum dots" takes place, and the same object changes to single electron device, for which the relevant parameters are related to the disorder through the barriers that separates the quantum dots, and the quasi-ballistic properties inside the quantum dots. The relevant parameters are then reduced to the scaling coefficient $\alpha$, the phase coherence 
length of the charge carriers, and the activation energy.

[1] see e.g. for Si nanostructures: A. T. Tilke et al., Prog. In Quantum Elec. 25 (2001) 97).

[2] Gareth J. Evans, Hiroshi Mizuta, and Haroon Ahmed, Jpn. J. Appl. Phys. 40,(2001) 5837.

[3] Tans S.J, Dekker C, Nature 404, 834 (2000).

[4] M. Bockrath, W. Liang, D. Bozovic, J.H. Hafner, C.M. Lieber, M. Tinkham and H. Park, Science 291, 283 (2001).

[5] Ania C. Bleszynski, Floris A. Zwanenburg, R. M. Westervelt, Aarnoud. L. Roest, Erik P. A. M. Bakkers, and Leo P. Kouwenhoven, Nano Letters 7 (2007) 2559

[6] T. Sakamoto, H. Kawaura and T. Baba, Appl. Phys. Lett., 72, 795 (1998).

[7] R. Augke, W. Eberhardt, C. Single, F. E. Prins, D. A. Wharam, and D. P. Kern, Appl. Phys. Lett. 76, 2065 (2000).

[8] M. Hofheinz, X. Jehl, M. Sanquer, G. Molas, M. Vinet and S. Deleonibus, Appl. Phys. Lett. 89, $143504(2006)$.

[9] A. Rochefort, D.R. Salahub, and P. Avouris, Chem. Phys. Lett 297,45 (1998).

[10] T. Hertel, R. Walkup, Ph. Avouris, Phys. Rev. B 58, 13870 (1998).

[11] A. Bezryadin, A.R.M. Verschueren, S.J. Tans and C. Dekker. Phys. Rev. Lett. 80, 4036 (1998).

[12] T. Hertel, R. Martel and Ph. Avouris. J. Phys. Chem. B 102, 910 (1998).

[13] Bachtold A, Henny M, Terrier C, Strunk C, Schoenenberger C, Salvetat JP, Bonard JM, and Forro L, Appl. Phys. Lett. 73, 274 (1998).

[14] M. Kruger, I. Widmer, T. Nussbaumer, M. Buitelaar and C. Schoenenberger, New J. Phys. 5, 138 (2003).

[15] Minot ED, Yaish Y, Sazonova V, Park JY, Brink M, and McEuen PL, Phys. Rev. Lett 90, $156401(2003)$.

[16] A. Bachtold, M. S. Fuhrer, S. Plyasunov, M. Forero, Erik H. Anderson, A. Zettl, and Paul L. McEuen, Phys. Rev. Lett. 84, 6082 (2000).

[17] T.L.Wade, and J.-E. Wegrowe, Eur. Phys. J. Appl. Phys. 29, 3 (2005).

[18] Physical Properties of Multiwall Nanotubes, L. Forro and C. Schoenenberger ,In: Carbon Nanotubes, edited by M.S. Dresselhaus, G. Dresselhaus and Ph. Avouris (Springer, Berlin Heidelberg, New York 2001). 
[19] Electrical Transport Through Single-Wall Carbon Nanotubes, Zhen Yao, Cees Dekker, and Phaedon Avouris, in: Book Carbon Nanotubes: Synthesis, Structure, Properties and Application , Topics Appl. Phys. 80, 147171 (2001) (M. S. Dresselhaus, G. Dresselhaus, Ph. Avouris (Eds.); Springer-Verlag Berlin Heidelberg 2001), and Ph. Avouris and Jia Chen Laterials Today, Vol 9, October 2006 pp 46-54.

[20] H. Dai, A. Javey; E. Pop, D. Mann, W. Kim, and Y. Lu, Nano, 1 (2006), 1 and rreference therein

[21] Understanding Carbon Nanotubes From Basics to Applications, Series: Lecture Notes in Physics, Vol. 677 Loiseau, A.; Launois, P.; Petit, P.; Roche, S.; Salvetat, J.-P. (Eds.) 2006.

[22] X. Hoffer, Ch. Klinke, J.-M. Bonard, L. Gravier, and J.-E. Wegrowe, Europhys. Lett., 67, 103 (2004).

[23] J.-F. Dayen , X. Hoffer, T. L. Wade, M. Konczykowski, and J.-E. Wegrowe, Phys. Rev. B 72 (7), $073402(2005)$.

[24] J.-F. Dayen,X. Jehl, T. L. Wade, M. Sanquer, and J.-E. Wegrowe, Phys. Stat. Sol.(b), 243, 3413 (2006).

[25] C. Schoenenberger, A. Bachtold, C. Strunk, J.-P. Salvetat, et L. Forro, Appl.Phys.A 69, 283 (1999).

[26] B. Stojetz, C. Hagen, C. Hendlmeier, E. Ljubovic, L. Forro, and C. Strunk, New J. Phys. 6, 27 (2004).

[27] N. Kang, J. S. Hu, W. J. Kong, L. Lu, D. L. Zhang, Z. W. Pan, and S. S. Xie, Phys. Rev. B 66, $241403(2002)$.

[28] J. Haruyama, I. Takesue, S. Kato, K. Takazawa, Y. Sato, App. Surf. Science, 175-176, 597 (2001).

[29] A. Bachtold et al., Nature 397673 (1999).

[30] J-O Lee et al 2000 Phys. Rev. B 61, 16362 (2001); Lee J-O 2001 Phys. Rev. B 64157402 (2001); C. Schoenenberger and A. Bachtold Phys. Rev. B 64157401.

[31] A. Fujiwara, K. Tomiyama, H. Suematsu, M. Yumura and K. Uchida, Phys. Rev. B 6013492 (1999).

[32] G. Fedorov, B. Lassagne, M. Sagnes, B. Raquet, J.-M. Broto, F. Triozon, S. Roche and E. Flahaut Phys. Rev. Lett. 94066801 (2005).

[33] T.H. Man and A.F. Morpurgo, Phys. Rev. Lett. 95026801 (2005). 
[34] S. Frank, Ph. Poncharal, Z.L. Wang and W.A. de Heer., Science 280, 1744 (1998).

[35] B. Stojetz, C. Miko, L. Forro and Ch. Strunk, Phys. Rev. Lett. 94186802 (2005).

[36] F. Triozon, S. Roche, A. Rubio and D. Mayou, Phys. Rev.B 69121410 (2004).

[37] J.-F. Dayen, A. Rumyantseva, C. Ciornei, C.S. Cojocaru, T. L. Wade, D. Pribat and J.-E. Wegrowe, Appl. Phys. Lett. 90, 173110 (2007)

[38] B.L. Altshuler, A.G. Aronov, and D.E. Khmelnitskii, J. Phys. C 15, 7367 (1982).

[39] B.L. Altshuler, A.G. Aronov: in Electron Electron Interactions in Disordered Systems, ed. by M. Pollak A.L. Efros (North-Holland, Amsterdam 1985);

[40] E. Akkermans, G. Montambaux, Mesoscopic Physics of Electrons and Photons, Cambdrige University Press, Cambridge (2007).

[41] M. S. Fuhrer, V. H. Crespi, M. L. Cohen and A. Zettl, Solid State Communications 109, 105 (1999).

[42] H. Grabert and M. H. Devoret, Single Charge Tunneling, Plenum Press, New York, Vol. 294 (1992).

[43] F. Pierre, H. Pothier, P. Joyez, N. O. Birge, D. Esteve, and M. H. Devoret, Phys. Rev. Lett. 86, 1590 (2001).

[44] J. Rollbuhler, H. Grabert, Phys Rev Lett. 87,126804 (2001).

[45] R. Egger, and A.O. Gogolin , Chem. Phys., 281, 447 (2002).

[46] A . Fontcuberta i Morral, J. Arbiol, J.D. Prades, A. Cirera, J.R. Morante, Adv. Mater. 19, 1347 (2007).

[47] J. Arbiol, B. Kalache, P. Roca i Cabarrocas, J. R. Morante, A. Fontcuberta i Morral, Nanotechnology 18 (2007) 305606.

[48] M. Bockrath, D. H. Cobden, J. Lu, A. G. Rinzler, R. E. Smalley, L. Balents, and P. L. McEuen, Nature 397, 598 (1999).

[49] Z. Yao, H.W. C. Postma, L. Balents, and C. Dekker, Nature (London) 402, 273 (1999).

[50] H.W. C. Postma, M. de-Jonge, and C. Dekker, Phys. Rev.B 62, R10 653 (2000).

[51] J. Nygard, D.H. Cobden, M. Bockrath, P.L. McEuen, and P.E. Lindelof, Appl. Phys. A. 297 (1999) and 84, $6082(2000)$.

[52] J. Haruyama, I. Takesue, and Y. Sato, Appl. Phys. Lett. 77, 2891 (2000).

[53] A. Bachtold, M. de Jonge, K. Grove-Rasmussen, P. L. McEuen, M. Buitelaar, and C. Schoenenberger, Phys. Rev. Lett. 87, 166801 (2001). 
[54] R. Tarkiainen, M. Ahlskog, J. Penttila, L. Roschier, P. Hakonen, M. Paalanen, and E. Sonin, Phys. Rev. B 64, 195412 (2001).

[55] A. Kanda, K. Tsukagoshi, Y. Aoyagi, and Y. Ootuka, Phys. Rev. Lett. 92, 036801 (2004).

[56] M. E. Gershenson, Yu. B. Khavin, A. G. Mikhalchuk, H. M. Bozler, and A. L. Bogdanov, Phys. Rev. Lett., 79, 725 (1997).

[57] Y. V. Nazarov, Phys. Rev.Lett., 82,1245 (1999).

[58] D. A. Bagrets and Yu. V. Nazarov, Phys. Rev. Lett. 94, 056801 (2005)

[59] D.S. Golubev and A.D. Zaikin, Phys. Rev. B 74, 245329 (2006)

[60] D.S. Golubev and A.D. Zaikin, Phys. Rev. B 70, 165423 (2004)

[61] S.V. Panyukov and A.D. Zaikin, Phys. Rev. Lett. 67, 3168 (1991)

[62] Yu. V. Nazarov, Phys. Rev. Lett. 82, 1245 (1999)

[63] D.A. Bagrets and Yu.V. Nazarov, Phys. Rev. Lett. 94, 056801 (2005)

[64] G.L. Ingold, Yu.V. Nazarov. In: Single Charge Tunneling, ed. by H. Grabert and M.H. Devoret, NATO ASI Series B 294 (Plenum Press, New York 1992) p. 21. 